\title{
Chirality of nuclear rotation
}

\author{
V.I.Dimitrov*, S. Frauendorf and F. Dönau \\ Department of Physics, University of Notre Dame, Notre Dame, IN 46556, USA \\ and Institute for Nuclear and Hadronic Physics, Research Center Rossendorf, PB \\ 5101 19, 01314 Dresden, Germany
}

It is shown that the rotating mean field of triaxial nuclei can break the chiral symmetry. Two nearly degenerate $\Delta I=$ 1 rotational bands originate from the left-handed and righthanded solution.

PACS Numbers: 21.10.-k, 23.20.Lv, 25.70.Gh, 27.60+j

Chirality appears in molecules composed of more than four different atoms and is typical for the biomolecules. In chemistry it is of static nature because it characterizes the geometrical arrangement of the atoms. Particle physics is the other field where chirality is encountered. Here it has a dynamical character, since it distinguishes between the parallel and antiparallel orientation of the spin with respect to the momentum of massless fermions. Meng and Frauendorf [1] recently pointed out that the rotation of triaxial nuclei may attain a chiral character. The lower panel of fig. 1 illustrates this new possibility. We denote the three principal axes $(\mathrm{PA})$ of triaxial density distribution by $\mathrm{l}$, i, and s, which stand for long, intermediate and short, respectively. The angular momentum vector $\vec{J}$ introduces chirality by selecting one of the octants. In four of the octants the axes l, i, and s form a left-handed and in the other four a right-handed system. This gives rise to two degenerate rotational bands because all octants are energetically equivalent. Hence the chirality of nuclear rotation results from a combination of dynamics (the angular momentum) and geometry (the triaxial shape).

Our symmetry argument is based on the presumption that the axis of uniform rotation needs not to agree with one of the PA of the density distribution. This does not hold for a rigid triaxial body, like a molecule for example, which can uniformly rotate only about the l- and s-axes. However, already Rieman [2] pointed out that a liquid may uniformly rotate about an axis tilted with respect to the PA, if there is an intrinsic vortical motion. In the case of the nucleus the quantization of the angular momentum of the nucleons at the Fermi surface generates the vorticity which enables rotation about a tilted axis.

The semiclassical mean field description of tilted nuclear rotation was developed in [3 5]. In the following we shall refer to it as the Tilted Axis Cranking (TAC) approach [5]. Fig. 1 illustrates the different symmetries

*Permanent adress: Faculty of Physics, University of Sofia, 1164 Sofia, Bulgaria if the mean field is assumed to be reflection symmetric. In the upper panel the axis of rotation (which is chosen to be z) coincides with one of the PA, i. e. the finite rotation $\mathcal{R}_{z}(\pi)=1$. This symmetry implies the signature quantum number $\alpha$, which restricts the total angular momentum to the values $I=\alpha+2 n$, with $n$ integer $(\Delta I=2$ band) [7]. In the middle panel the rotational axis lies in one of the planes spanned by two PA (planar tilt). Since then $\mathcal{R}_{z}(\pi) \neq 1$, there is no longer a restriction of the values $I$ can take. The band is a sequence of states the $I$ of which differ by $1(\Delta I=1$ band). There is a second symmetry in the upper two panels: The rotation $\mathcal{R}_{y}(\pi)$ transforms the density into an identical position but changes the sign of the angular momentum vector $\vec{J}$. Since the latter is odd under the time reversal operation $\mathcal{T}$, the combination $\mathcal{T R}_{y}(\pi)=1$.

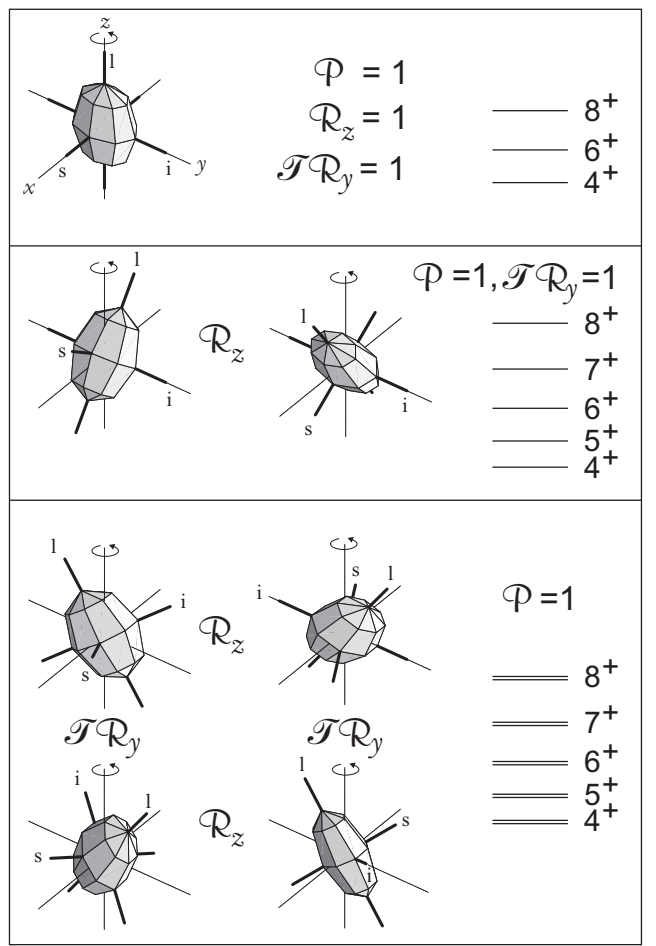

FIG. 1. The discrete symmetries of the mean field of a rotating triaxial reflection symmetric nucleus (three mirror planes). The axis of rotation ( $\mathrm{z}$ ) is marked by the circular arrow. It coincides with the angular momentum $\vec{J}$. The structure of the rotational bands associated with each symmetry type is illustrated on the right side. Note the change of chirality induced by $\mathcal{T} \mathcal{R}_{y}(\pi)$ in the lowest panel. 
In the lower panel the axis of rotation is out of the three planes spanned by the PA. The operation $\mathcal{T R}_{y}(\pi) \neq 1$. It changes the chirality of the axes $1, \mathrm{i}$ and $\mathrm{s}$ with respect to the axis of rotation $\vec{J}$. Since the left- and the right-handed solutions have a the same energy, they give rise to two degenerate $\Delta I=1$ bands. They are the even $(|+\rangle)$ and odd $(|-\rangle)$ linear combinations of the two chiralites, which restore the spontaneously broken $\mathcal{T R}_{y}(\pi)$ symmetry.

Ref. 11] studied a proton particle and a neutron hole coupled to a triaxial rotor with maximal asymmetry $\left(\gamma=30^{\circ}\right)$ and the irrotational flow relation $\mathcal{J}_{l}=\mathcal{J}_{s}<\mathcal{J}_{i}$ between the moments of inertia. The solutions of this model case describe the dynamics of the orientation of $\vec{J}$. It was found that at the beginning of the bands the angular momentum originates from the particle and the hole, whose individual angular momenta are aligned with the s- and l-axes. These orientations correspond to a maximal overlap of the particle and hole densities with the triaxial potential. A $\Delta I=1$ band is generated by adding the rotor momentum $\vec{R}$ in the s-l plane (planar tilt). There is a second $\Delta I=1$ band representing a vibration of $\vec{J}$ out of the s-l plane. Higher in the band, $\vec{R}$ reorients towards the i-axis, which has the maximal moment of inertia. The left- and the right-handed positions of $\vec{J}$ separate. Since they couple only by some tunneling, the two bands come very close together in energy.

The model study assumed: i) a triaxial shape with $\gamma \approx 30^{\circ}$, ii) a combination of excited high-j protons and high-j neutron holes and iii) $\mathcal{J}_{l}=\mathcal{J}_{s}<\mathcal{J}_{i}$. In order to decide if i)-iii) are simultaneously realized in actual nuclei we developed a 3D cranking code, which permits a microscopic study. In essence, it is the mean field approximation to the two body Routhian $H^{\prime}=t+v_{12}-\omega \hat{J}_{z}$, where any orientation of the deformed average potential $V_{d e f}$ is permitted (cf. [3 [5]). Only reflection symmetry is demanded. In the actual calculation the standard shell correction approximation is used (cf. [8]). The quasi particle Routhian

$$
\begin{array}{r}
h^{\prime}=h_{s p h}+V_{d e f}(\varepsilon, \gamma)-\Delta\left(P+P^{+}\right)-\lambda N \\
-\omega\left(\hat{J}_{1} \sin \vartheta \sin \varphi+\hat{J}_{2} \sin \vartheta \cos \varphi+\hat{J}_{3} \cos \vartheta\right)
\end{array}
$$

is diagonalized. The monopole pair field $\Delta\left(P+P^{+}\right)$ and the term $\lambda N$, which controls the particle number $N$, are understood as sums of the neutron and proton parts. This yields quasi-particle energies and states, from which the the quasi particle configuration $\mid \omega, \varepsilon, \gamma, \vartheta, \varphi>$ is constructed. The spherical Woods-Saxon energies 9] are used for $h_{s p h}$ and the Nilsson model [8] for $V_{d e f}(\varepsilon, \gamma)$. In a separate paper [10] we demonstrate that this hybrid approximates well the deformed Woods Saxon potential, which provides a good description of the rotational spectra of the region around mass $A=135$.

The total Routhian is calculated by means of the shell correction method,

$$
\begin{aligned}
E^{\prime}(\omega, \varepsilon, \gamma, \vartheta, \varphi)= & <\omega, \varepsilon, \gamma, \vartheta, \varphi\left|h^{\prime}\right| \omega, \varepsilon, \gamma, \vartheta> \\
- & <\omega=0, \varepsilon, \gamma\left|h^{\prime}\right| \omega=0, \varepsilon, \gamma> \\
& +E_{L D}(\varepsilon, \gamma)-E_{\text {smooth }}(\varepsilon, \gamma)
\end{aligned}
$$

where $E_{\text {smooth }}$ and $E_{L D}$ are, respectively, the Strutinsky averaged and liquid drop energies at $\omega=0$. The method is described for example in [8]. The deformation parameters $\varepsilon$ and $\gamma$ and the tilt angles $\vartheta$ and $\varphi$ are found for a given frequency $\omega$ and fixed configuration by minimizing the total Routhian $E^{\prime}$. The value of $\Delta$ is kept fixed at $80 \%$ of the experimental odd-even mass difference for the nucleus of interest. The values of $\lambda_{p}$ and and $\lambda_{n}$ are adjusted to have the correct values of $\langle Z\rangle$ and $\langle N\rangle$ for $\omega=0$ and kept fixed for $\omega>0$.

The electro-magnetic transition matrix elements for the left- and right-handed solutions are obtained by means of the straightforward generalization of the semiclassical expressions given in [1,5,5]. If tunneling is negligible there are no matrixelements between the two TAC states $\mid \omega>$ and $\mathcal{T} \mathcal{R}_{y}(\pi) \mid \omega>$, because the electromagnetic field cannot provide the amount of angular momentum which is necessary to reorient the long vector $\vec{J}$. Then the probabilities for the transitions $+\rightarrow+$ and $-\rightarrow-$ are given by the mean value and the ones for $+\rightarrow-$ and $-\rightarrow+$ by half the difference of the transition matrix elements of the left- and the right-handed solutions. We assume zero tunneling.

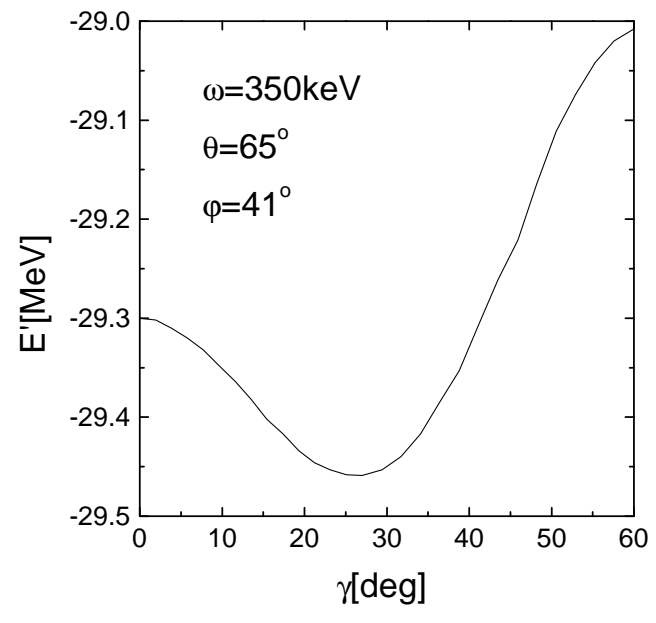

FIG. 2 . Total Routhian $E^{\prime}\left(\omega=0.35 \mathrm{MeV}, \varepsilon=0.175, \vartheta=65^{\circ}, \varphi=41^{\circ}\right)$ as function of the triaxiality parameter $\gamma$.

We investigated the quasi particle configuration $\left[\pi h_{11 / 2}, \nu h_{11 / 2}\right]$ in ${ }_{59}^{134} \operatorname{Pr}_{75}$, which was suggested as a candidate for chiral rotation [1]. The microscopic calculations, presented in figs. 2- 4 as well as in the table, confirm the mechanism for the chirality as described in the model study [1]:

- The shape is triaxially deformed with $\gamma$ close to $30^{\circ}$ (cf. Fig. 2). The deformation remains nearly the same within the frequency range $0.3 \mathrm{MeV} \leq \omega \leq 0.5 \mathrm{MeV}$ studied. 
-The $h_{11 / 2}$ quasi proton has particle character and tends to align with the s-axis. The $h_{11 / 2}$ quasi neutron has hole character and prefers the l-axis.

-The moment of inertia $\mathcal{J}_{i}$ is the largest. Accordingly, the orientation of $\vec{J}$ ( position of the minimum in the $\vartheta-\varphi$ plane) moves from the s-l plane $\left(\vartheta=60^{\circ}, \varphi=0^{\circ}\right)$ towards the i-axis $\left(\vartheta=90^{\circ}, \varphi=90^{\circ}\right)$. Fig. 3 shows the energy in $\vartheta-\varphi$ plane at $\omega=0.35 \mathrm{MeV}$ when the orientation is furthest out in the chiral sector. The minimum is soft in $\varphi$ direction.

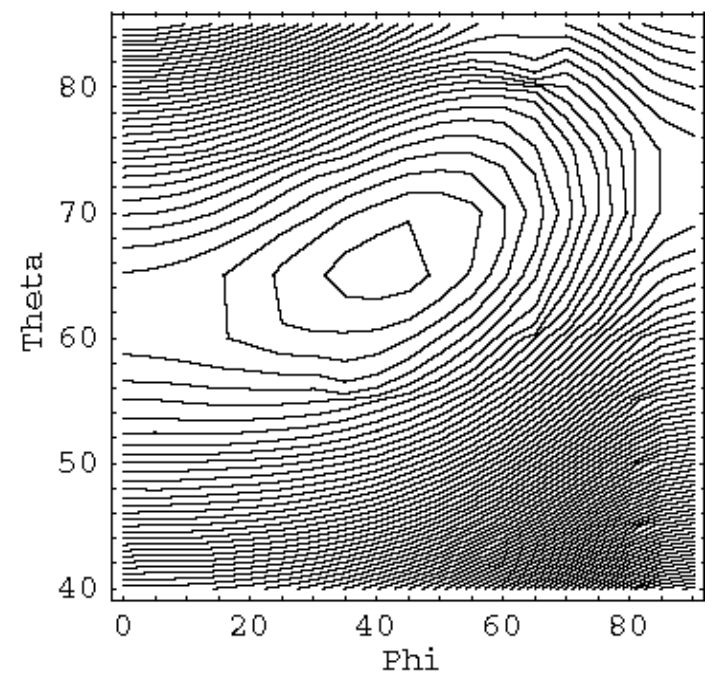

FIG. $3 . \quad$ Total Routhian $E^{\prime}\left(\omega=0.35 \mathrm{MeV}, \varepsilon=0.175, \gamma=27^{\circ}\right)$ as function of tilt angles $\theta$ and $\varphi$. The energy difference between the contour lines is $18 \mathrm{keV}$. A cylindric projection is used. The PA correspond to $l: \vartheta=90^{\circ} ; i: \vartheta=90^{\circ}, \varphi=90^{\circ} ; s: \vartheta=0^{\circ}, \varphi=0^{\circ}$

The TAC mean field approach treats the orientation of the rotational axis in a static way. One can only give a simple interpretation of the two distinct cases of well conserved $\mathcal{T} \mathcal{R}_{y}(\pi)$-symmetry (two separated $\Delta I=1$ bands) and strongly broken $\mathcal{T R}_{y}(\pi)$-symmetry (two degenerate $\Delta I=1$ bands). For $\omega<0.3 \mathrm{MeV}, \varphi=0$, i. e. the rotational axis lies in the s-l plane. For $\omega=0.3 \mathrm{MeV}$, the barrier at $\varphi=0$ is only a few $k e V$. Accordingly, two separate $\Delta I=1$ bands are expected, which correspond to the first two vibrational states of the angular momentum vector $\vec{J}$. For $\omega=0.325-0.375 \mathrm{MeV}$, the rotation is chiral and the two bands come close. Strong degeneracy is not expected, because the barrier at $\varphi=0$ is only $60 \mathrm{keV}$. For larger $\omega$, the minimum moves slowly towards the i-axis $\left(\vartheta=90^{\circ}, \varphi=90^{\circ}\right)$. Fig. 4 shows the experimental energies of two lowest $\Delta I=1$ bands of positive parity, which were suggested to form a chiral pair [1]. Their relative position correlates with the calculation: At $I=10$, there is a substantial splitting between them, which becomes very small around $I=15$, and remains small for higher $I$.

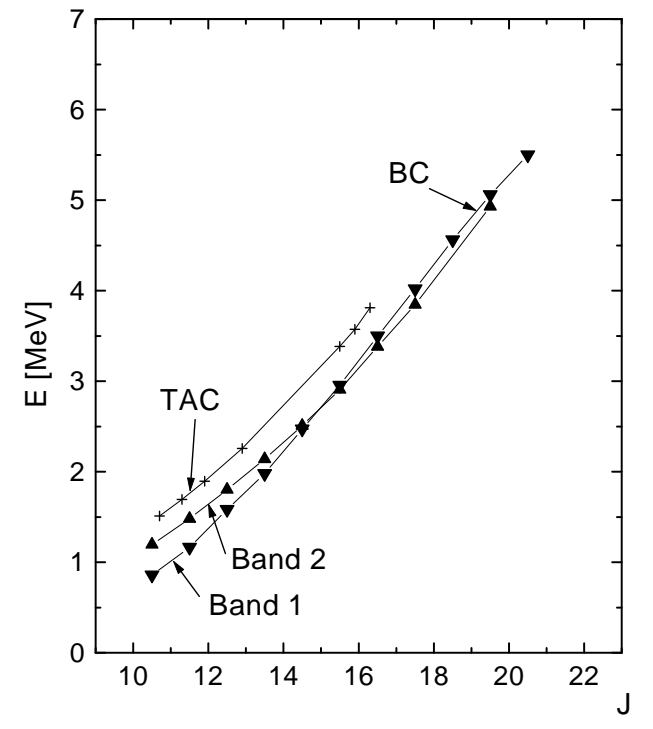

FIG. 4. Energy of the chiral bands in ${ }_{59}^{134} \operatorname{Pr}_{75}$. Triangles: Experimental energies [11] of the two bands assigned to the configuration $\left[\pi h_{11 / 2}, \nu h_{11 / 2}\right]$ vs. $J=I+1 / 2$. Crosses: TAC calculation (the zero point is arbitrary) .

For given $\omega$, band 1 has about two units of angular momentum more than band 2 (cf. fig. $6 \mathrm{~b}$ in [11]). The difference indicates substantial tunneling, which is expected because the calculated energy barrier between the two solutions is less than $100 \mathrm{keV}$ in $\varphi$ direction and the depth of the energy minimum with respect to the triaxiality parameter $\gamma$ is only $180 \mathrm{keV}$. The situation is similar to the weak breaking of the reflection symmetry in the light actinide nuclei (see [13]). There, the barrier between the two reflection symmetric solutions is calculated to be few $100 \mathrm{keV}$. The two bands of opposite parity, which should be

degenerate for strongly broken reflection symmetry, come fairly close in energy. But usually there is a difference between the angular momenta at given frequency

which is of the order of $1-2$ units in most of the observed frequency range (1.3 for $\left.{ }^{226} \mathrm{Th}\right)$. Since the TAC mean field calculations do not include tunneling they must be compared with the averages over the two chiral sister-bands. The table demonstrates that the calculated curve $J(\omega)$ reproduces quite well the average $\bar{J}(\omega)$ of the two experimental curves.

Around $J=14$, the calculated ratio of $\left.B(M 1) / B(E 2) \approx 3\left(\mu_{N}\right)^{2} /(e b)^{2}\right]$ for intraband transitions is consistent with the mean of the experimental values of $\left.5 \pm 3\left(\mu_{N}\right)^{2} /(e b)^{2}\right]$ [11]. The experimental branching ratios $B\left(M 1, I_{2} \rightarrow I_{1}-1\right) / B\left(M 1, I_{2} \rightarrow\right.$ $\left.I_{2}-1\right)=0.1,0.1,0.8,0.5$ for $I=13,14,14,16$ 11] correlate with the calculated increase of the interband M1 transition probability when $\varphi$ becomes large. The experimental ratios for lower $I$ cannot be compared with the TAC calculations because the underlying assumption of no tunneling between the left- and righthanded solutions does not hold for small $\varphi$. The data does not permit us checking the characteristic small ra- 
tios $B\left(E 2, I_{2} \rightarrow I_{1}-2\right) / B\left(E 2, I_{2} \rightarrow I_{2}-2\right)$.

In the calculation, the one quasi neutron configuration $\left[h_{11 / 2}\right]$ is crossed by the three quasi neutron configuration $\left[h_{11 / 2}^{3}\right]$ near $\omega=0.4 \mathrm{MeV}$. We ignore this crossing ("BC-crossing" in the common letter convention []]), showing the results for the $\left[h_{11 / 2}\right]$ configuration. The reason is that in experiment the crossing is observed at the higher frequency of $\omega=0.5 \mathrm{MeV}$ [11]. This kind of delay appears systematically in configurations with protons and neutrons in the same $\mathrm{j}$-shell and is not accounted for by the Cranking model (see [12] and the literature cited therein).

As a second example for chirality, we found the configuration $\left[\pi i_{13 / 2}, \nu i_{13 / 2}\right]$ in ${ }_{77}^{188} \operatorname{Ir}_{111}$ (cf. table). The equilibrium shape is $\varepsilon=0.21$ and $\gamma=40^{\circ}$. Like for ${ }^{134} \mathrm{Pr}$ we encountered the BC-crossing, which leads to the planar solution at $\omega=0.3 \mathrm{MeV}$. The existence of a chiral solution based on the excited configuration $\left[\nu i_{13 / 2}\right]$, analogous to the one based on $\left[\nu h_{11 / 2}\right]$ in ${ }^{134} \mathrm{Pr}$, is likely but we did not calculate it. Ref. [4] found a chiral angular momentum geometry for the configuration $\left[\pi g_{9 / 2}, \nu g_{9 / 2}\right]$ in ${ }_{39}^{84} \mathrm{Y}_{45}$ assuming a triaxial shape with $\gamma=30^{\circ}$. We calculated for this configuration an axial equilibrium shape with $\varepsilon=0.19, \gamma=60^{\circ}$ and $\vartheta=90^{\circ}$. This result is consistent with the observation of an even- $I$ yrast band (case of good $\mathcal{R}_{z}(\pi)$-symmetry, upper panel of fig. 1).

In conclusion: We demonstrated the existence of selfconsistent rotating mean field solutions with chiral character for ${ }^{134} \mathrm{Pr}$ and ${ }^{188} \mathrm{Ir}$. The left- and right-handed solutions give rise to two near degenerate $\Delta I=1$ bands of the same parity with supressed electric quadrupole transitions between them. A pair of bands with the expected properties is observed in ${ }^{134} \mathrm{Pr}$. The calculated small energy gain due to breaking of the chiral symmetry is in in accordance with the experiment, which points to substantial tunneling beween the two solutions of opposite chirality.

This work was supported by the DOE grant DE-FG0295ER40934.

[1] S. Frauendorf, and Meng, J., 1997, Nucl. Phys. A 617, 131 (1997)

[2] Riemann, B., Abh. Kön. Ges. Wiss. (Göttingen) 9 (1860) 1

[3] Kerman, A.K., and Onishi, N., Nucl. Phys. A 361 ( 1981) 179

[4] Frisk, H., and Bengtsson, R., Phys. Lett. 196B (1987) 14

[5] S.Frauendorf, Nucl. Phys. A557 (1993) 259c

[6] S. Frauendorf, Z. Phys. A385, 163 (1997)

[7] Bengtsson, R., and Frauendorf, S., Nucl. Phys. A 327 (1979) 139
[8] Shapes and Shells in Nuclear Structure, S.G. Nilsson and I. Ragnarsson, Cambridge University Press, 1995

[9] S.Cwiok et al., Comp.Phys.Comm. 46 (1987) 379

[10] V.I.Dimitrov et al., Phys. Rev. C, submitted

[11] C. Petrache et al. , Nucl. Phys. A 597 (1996) 106

[12] S.Frauendorf and J Sheikh, Nucl. Phys. A 645 (1999) 509

[13] P. A. Butler, W. Nazarewicz, Rev. Mod. Phys. 68 (1996) 349

\begin{tabular}{lllllllll}
\hline \hline$\omega[M e V]$ & $\vartheta$ & $\varphi$ & $J$ & $\bar{J}$ & \multicolumn{3}{c}{$B(M 1)\left[\left(\mu_{N}\right)^{2}\right]$} & \multicolumn{2}{l}{$B(E 2)\left[0.1(e b)^{2}\right]$} \\
\hline 0.250 & 60 & 0 & 10.2 & & 1.29 & 0.00 & 0.67 & 0.00 \\
0.300 & 60 & 21 & 10.7 & 10.9 & 1.16 & 0.13 & 0.91 & 0.08 \\
0.325 & 62 & 33 & 11.3 & 11.5 & 0.96 & 0.30 & 1.50 & 0.13 \\
0.350 & 65 & 41 & 11.9 & 12.2 & 0.80 & 0.41 & 2.11 & 0.13 \\
0.375 & 70 & 54 & 12.9 & 13.0 & 0.54 & 0.52 & 3.02 & 0.08 \\
0.400 & 75 & 66 & 14.4 & 13.6 & 0.35 & 0.57 & 3.96 & 0.03 \\
0.450 & 75 & 65 & 15.5 & 14.8 & 0.37 & 0.41 & 3.90 & 0.03 \\
0.475 & 75 & 68 & 15.9 & 15.3 & 0.37 & 0.34 & 4.06 & 0.02 \\
\hline 0.200 & 66 & 13 & 12.2 & & 1.43 & 0.23 & 1.35 & 0.16 \\
0.225 & 66 & 25 & 12.7 & & 1.25 & 0.83 & 2.29 & 0.53 \\
0.250 & 66 & 32 & 13.4 & & 1.12 & 1.23 & 3.27 & 0.74 \\
0.275 & 72 & 44 & 14.7 & & 0.60 & 2.13 & 6.55 & 0.53 \\
0.300 & 85 & 59 & 20.2 & & 0.04 & 3.49 & 1.12 & 0.03
\end{tabular}

TABLE I. Orientation angles $\vartheta, \varphi$ and value $J$ of the angular momentum and reduced transition probabilites of the configuration $\left[\pi h_{11 / 2}, \nu h_{11 / 2}\right]$ in ${ }_{59}^{134} \operatorname{Pr}_{75}$ (upper part) and of the configuration $\left[\pi i_{13 / 2}, \nu i_{13 / 2}\right]$ in ${ }_{77}^{188} \operatorname{Ir}_{111}$ (lower part) as calculated by TAC. Chirality corresponds to both $\vartheta \neq 0^{\circ}$ and $90^{\circ}$ and $\varphi \neq 0^{\circ}$ and $90^{\circ}$. The experimental values $\bar{J}(\omega)=\left(I_{1}(\omega)+I_{2}(\omega)\right) / 2+1 / 2$, where $I_{1,2}(\omega)$ are obtained by interpolating between the discrete points for band 1 and 2 , respectively. For the transition probabilities the left column gives the intraband transitions $(+\rightarrow+$ and $-\rightarrow-)$ and the right the interband transitions $(+\rightarrow-$ and $-\rightarrow+)$. When the chiral symmetry is conserved, the intraband probabiites are equal to the non zero value. 